\title{
Identification and valuing the Spanish fortification in Algeria Case of the town of Bejaia
}

\section{Amina Korichi}

Université Mouloud Mammeri, département d'architecture, Tizi-Ouzou, Algérie. korichi.pg2009@yahoo.fr

\begin{abstract}
Military architecture has shaped the landscape of most Algerian towns since the dawn of time. It therefore contains an exceptional heritage related to military and defense activities. Nowadays, this legacy with its valuable shapes and traces, suffers unfortunately from a lack of recognition and is abandoned and poorly reused. Bejaia, like all Algerian coastal cities, conceals an exceptional field in military-defensive historic buildings. The typological diversity and values they carry, from the Roman occupation (33BC) to the advent of French occupation in 1833, have provided to this multi-thousandyear-old city a defensive system made of typological transformation and stratification of defensive system. In this communication, we want to present and illustrate the state of conservation and enhancement of the defensive system of the Spanish era, through the case of the fortifications of the city of Bejaia, strongly marked by the construction of fort imperial (bordj Moussa), and reconstruction of the citadel of the city.
\end{abstract}

Keywords: identification, defensive system, Spanish, Bejaia.

\section{Introduction}

Le paysage urbain que nous offrent les villes Algériennes notamment la ville de Bejaia semble a priori, être le résultat d'une évolution faite de juxtaposition et superposition de plusieurs cultures urbaines relatives aux forces dominantes à un moment ou un autre des lieux. Les Carthaginois, les Romains, les Vandales, les dynasties berbéro-musulmanes (Hammadides, Almohades et Hafsides), les espagnols, les ottomans puis les français ont marqué l'espace urbain. Cette emprunte est extrêmement lisible sur le système défensif qui orne la structure urbaine de Bejaia.

A la fin du XVème siècle, la reconquête chrétienne de l'Andalousie a permis à l'Espagne d'occuper de nombreux sites et ports de la côte algérienne : Mers el-Kébir, Oran, Bejaia et l'îlot du Penon en face d'Alger, et même d'imposer des tributs à d'autres villes (Khelifa, 2007).
L'emplacement stratégique de la ville de Bejaia et sa prospérité attirent la convoitise des espagnols, le comte Pedre Navarre qui prend Bougie le 5 Janvier 1509, ils la nommèrent Buggia, pour une durée de 45 ans (Epalza, 1988). Avec leur arrivée, le périmètre urbain a été réduit à $1 / 3$ de l'enceinte romaine, et les $2 / 3$ sont délaissés et abandonnés pour des raisons économiques et défensives. Les Espagnoles détruisirent le minaret du château de la perle et le château de l'étoile et embarquèrent tous les objets de valeur comme les colonnes, les faïences et les objets en bois gravés contenus dans les deux bâtiments pour les emmener en Espagne (Dehabsourg, 1999). La ville se trouve dans un état ruiné et dépeuplé.

Après leur installation, Pierre de Navarre, Maitre de la ville de Bougie, s'occupa immédiatement à la fortifier. Il mit une garnison dans l'ancien château Vergelete (fort Abd El Kader), fit 
construire le château impérial (bordj Moussa), et jeta les fondements d'une nouvelle casbah sur le bord de la mer (Feraud , 1868). D'après Léon Africain, Pedro Navarro a fait bâtir une forteresse près de la mer, à un endroit où il existe une bonne plage, et a fortifié une vielle citadelle, également voisine de la mer à côté de l'arsenal (valérien , 2000). La nouvelle enceinte forme un triangle dont la base était la mer, elle reliait le fort Abd El Kader à la Casbah. L'enceinte longe les deux contreforts bridja et Moussa, dont le sommet du triangle sera le fort Barral (fort Moussa ou le grand château).

\section{La citadelle}

Construite à l'extrémité d'une anse sur les assises du vieux port romain. La casbah est située au Sud- Ouest de la ville dominant ainsi toute la baie. C'est le fort le plus important de la ville à la fois par sa surface, son volume et sa proximité du port. Sa forme est presque rectangulaire de 155 mètres de long sur 70 mètres de large.

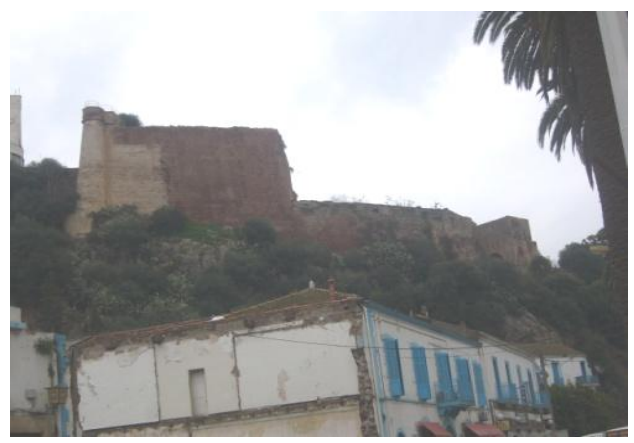

Fig.1-vue sur la casbah de Bejaia (Korichi.Amina 2010)

Cette imposante fortification héritée du passé reste un mystère pour les chercheurs qui s'y sont intéressé. Plusieurs confusions ont été relevées dans les écrits quant à la date de fondation de la citadelle. Selon Léon L'Africain, la citadelle fut construite à l'époque almohade. Le plan le plus ancien de la casbah date de 1539(Epalza , 1988), et provient des archives espagnoles, représente un projet de réforme d'une citadelle existante qui pouvait être la casbah almohade. Le dessin montre une double enceinte fortifiée projetée par Libran ((Epalza , 1988) sur la base de deux plans polygonaux à grandes dimensions, dont l'extérieur serait hexagonal et l'intérieur quadrangulaire, avec des bastions rhomboïdes défendus par des courtines à talus prononcés. Les murs extérieurs ont une hauteur de 35 pieds, et une épaisseur de 10 pieds (Epalza , 1988). Les bastions sont recouverts par des voûtes qui permettent l'installation de batteries.

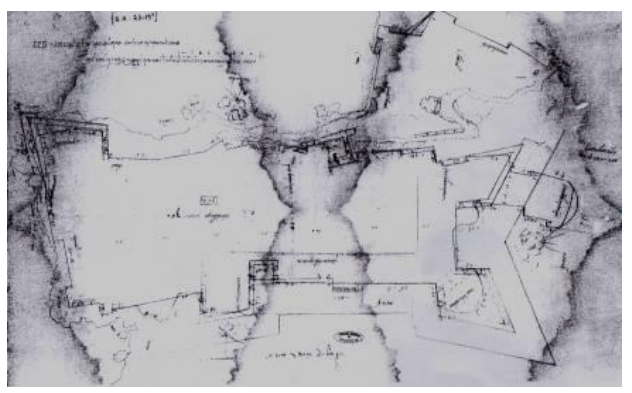

Fig.2- plan de la citadelle en 1535 (Epalza 1988)

Un rapport du Génie militaire sur la place de Bougie en 1833(Mahindad, 2002), confirme que la partie inférieure de la casbah était de diverses époques islamiques, par contre la partie supérieure était l'œuvre des espagnols.

Occupée par les espagnols pendant 45 ans, la casbah fut récupérée par les ottomans, qui en firent le siège de leur gouvernement. Les modifications apportées par les ottomans étaient essentiellement la reconstruction de l'ancienne mosquée, et la consolidation du rempart par la construction d'un bastion carré sur la partie basse, doté de merlon et couronné par des pyramidions.

A l'arrivée des français, la casbah a subi des dommages à cause de la nouvelle artillerie. Les remparts et la tour qui font face à la ville ont été considérablement rasés en 1853, les meurtrières et les clochetons ont par conséquent disparu.

Après leur installation, les français ont procédé à la réhabilitation de la citadelle à des fins militaires.

Après l'indépendance, la casbah se trouve dans un état de délabrement très poussé et plus particulièrement la mosquée (Khelifa, 1970). Une première étude a été lancée en 1978, afin de proposer un plan de sauvegarde et de restauration de la casbah. Cette étude a été vaine 
vue la non -disponibilité de la documentation (Zitouni, 1978). En 1980, suite à la demande du gouvernement algérien L' UNESCO, envoie son consultant pour élaboration d'un plan de sauvegarde de l'ancienne ville de Bejaia. Ce dernier nous donne une description détaillée de la casbah et de son état en 1980.

\subsection{Le fort}

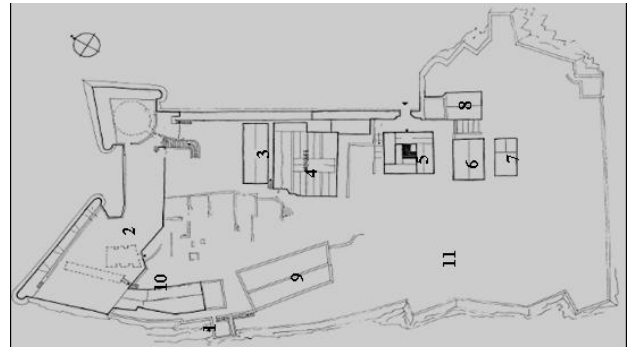

Fig.3- plan de la casbah, (Korichi. Amina 2011) Légende : 1 : Bâb dar essenaa, 2 : le fort espagnol, 3: bâtiment en mauvais état. 4 : la mosquée, 5 : maison a patio, $6:$ manutention, $7:$ artillerie, $8:$ artillerie, 9 : reste archéologique, 10 : Magasin, 11 : espace boisé.

C'est la masse la plus imposante qui domine la Casbah, édifié sous l'occupation espagnole. Cet ouvrage bastionné est flanqué de trois hautes et massives tours garnies de meurtrières. Ces tours ressemblent à d'énormes couleuvrines plantées en terre par la culasse (Feraud, 1858). Le fort renferme deux grandes salles rectangulaires voûtées. A partir des escaliers intégrés à ce fort, on atteint les terrasses et le chemin de ronde du rempart.

\subsection{Les bastions de la partie basse}

Ce sont des bastions conçus sur les restes du rempart Hammadite. Le premier bastion qui donne sur l'ancienne ville et le port, est un simple carré, couronné de merlons en forme de pyramide. La construction de ce bastion remonte probablement à l'époque almohade. L'utilisation des pyramidions pour couronner les merlons confirme cette hypothèse (Korichi, 2011). Le deuxième bastion de la partie basse a été édifié par les français au sud ouest de la citadelle, il referme dans sa partie basse une citerne.

\subsection{La Mosquée}

La mosquée est la construction la plus récente; elle fut édifiée en (1791) par ordre de Moustafa Pacha. C'est la grande mosquée sous la domination ottomane (Féraud, 1858). Sa conception, sur un plan rectangulaire, est très classique. Elle est constituée de quatre travées longitudinales et cinq transversales. Son axe principal est coiffé de trois coupoles octogonales. Son ordonnance intérieure et ses proportions, avec ses arcs outrepassés, sont encore de grande qualité malgré les dégradations et les modifications apportées à la partie centrale où les arcs ont été agrandis pour permettre la construction d'un plancher intermédiaire à l'époque coloniale.

\subsection{Les autres bâtiments}

Ce sont des Petits bâtiments de forme carrée. La première maison en face de l'entrée principale est d'origine espagnole (Herrmann, 1980), fortement transformée lors de l'occupation ottomane et française.

\subsection{Les portes}

La casbah est dotée de deux portes, une principale qui donne sur l'ancienne ville, et une autre qui donne sur la plaine appelée Bâb dar Essenaa.

La porte principale est une porte non flanquée percée dans l'épaisseur du rempart extérieur, composée de trois arcs en plein- cintre, surmontée d'un chemin de ronde, et décorée par deux colonnes qui supportent un fronton (Korichi, 2011).

La deuxième porte (Bâb dar essaneaa) est une porte percée dans une tour, composée de trois arcs en plein-cintre. La tour se développe en trois niveaux, le premier niveau où se trouve l'issue de la porte, est un passage voûté. Le deuxième niveau est une salle carrée dotée de meurtrière sur la face qui se trouve au dessus de la porte. Le troisième niveau est une terrasse accessible. 


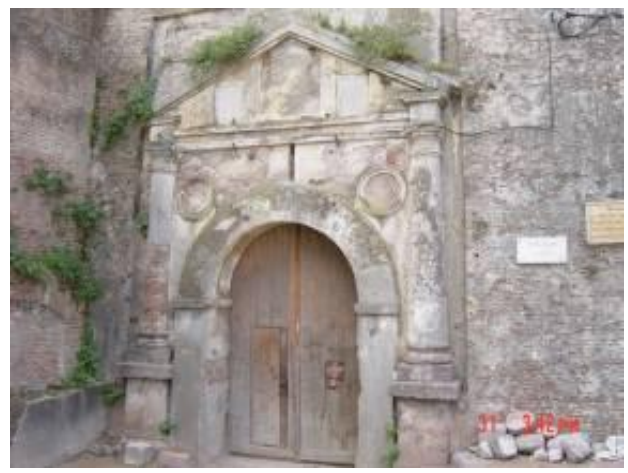

Fig. 4- vue sur la porte de la casbah (Korichi, Amina, 2010)

Aujourd'hui, la casbah se compose de plusieurs bâtiments témoins de différentes périodes, on trouve:

Les quatre bastions tels qu'ils sont décrits auparavant.

La mosquée restaurée pendant les années 90, réutilisée actuellement comme annexe de la bibliothèque nationale.

Plusieurs bâtiments datant de la période coloniale (une ancienne boulangerie, un dépôt d'artillerie et un hangar).

Des citernes pouvant contenir 200.000 litres d'eau, et des casemates

Des écuries partiellement détruites.

Des vestiges archéologiques dont la datation reste indéfinie.

Concernant les matériaux utilisés, la casbah est construite dans sa totalité en briques rougeâtres, néanmoins dans quelques parties, on trouve le moellon et la pierre.

\section{Fort Moussa}

Le grand château ou le château impérial à l'époque espagnole, Bordj Moussa à l'époque ottomane, fort Barral à l'époque française.

Le fort se trouve dans l'ancienne entité de Bejaia à proximité des deux anciens quartiers Bâb Elouz et Karramane. Il occupe un site exceptionnel qui domine toute la ville à l'exception du ravin de Bridja qui s'échappe à son champ de vision. Édifié par les espagnols durant la première moitié du 16ème siècle, sur les traces du palais Hammadite «de l'étoile» et probablement sur l'emplacement de ruines d'une bâtisse militaire romaine (korichi, 2011).

L'appellation bordj Moussa vient de la bataille rude entre kabyles, espagnols, et les sept valeureux guerriers (R'djel Essabaâ) qui prirent l'initiative de se sacrifier en s'introduisant dans l'enceinte du fort, où ils furent exécutés tous les sept par les soldats espagnols. Le premier de sept combattants portait le prénom de Moussa, d'où dérive donc le nom de bordj Moussa attribué par les Turcs à ce fort en reconnaissance au premier homme qui a osé s'aventurer dans le camp de l'ennemi.

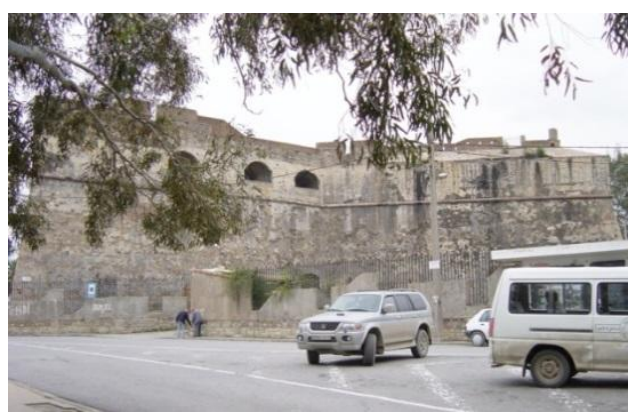

Fig. 5- Vue sur le bordj Moussa ( Korichi, Amina 2009)

Le plan du rez-de-chaussée fut envoyé au prince Philipe par le gouverneur don Luis de Peralta (Epalza, 1988). Ce plan dessiné par Librano, est un plan rectangulaire avec des bastions latéraux. Le fort a de gros murs à talus dont la hauteur est de 100 pieds et l'épaisseur de 35 pieds, avec douze ouvertures pour les batteries (Epalza, 1988). L'intérieur $\mathrm{du}$ fort a trois nefs longitudinales dont la centrale est la plus haute que les deux autres. Elle est aussi divisée en trois corps, grâce à quatre gros piliers adossés au mur extérieur. Les piliers supportent trois voûtes. A l'extrémité de la nef, se trouvent une chapelle et un escalier qui monte au premier étage.

La première description architecturale de ce fort était transcrite à l'occupation française à travers les rapports du génie militaire en vue de son entretient (Ministère de la guerre, 1838). D'après cette description, le bordj est une gigantesque construction, avec des murs inclinés. Le rez-de- 
chaussée était composé de trois grandes voûtes, de neufs mètres de hauteur et de six mètres de largeur. Ses murs étaient très épais tout particulièrement ceux du Nord qui donnait sur la montagne avec une épaisseur de six mètres. Les parements intérieurs et extérieurs étaient en maçonnerie de briques, ils étaient percés par des embrasures.

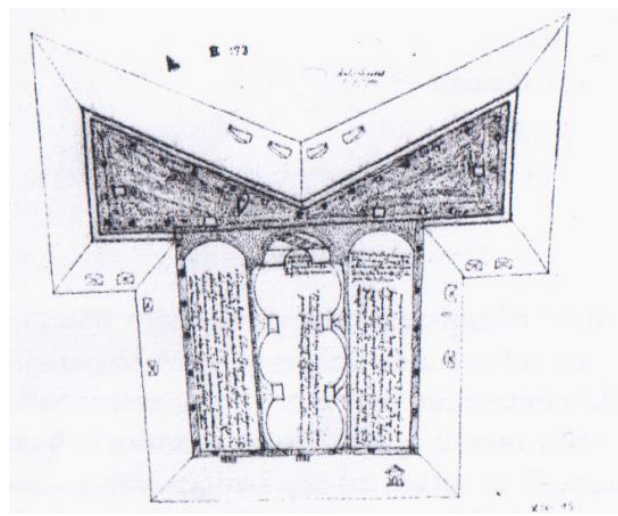

Fig. 6- plan du bordj Moussa à l'époque espagnole (Epalza, 1988)

La plate- forme était une vaste esplanade comprenant presque toute la surface du fort, elle était entourée des côtés sud, Est et ouest par une murette de quarante centimètres d'épaisseur et cent cinquante centimètres de hauteur. Sur toute sa surface, la plate -forme était percée par six évents. Le cavalier était du côté nord de la plate forme, il était fermé par un mur de 50 centimètres d'épaisseur. Au coin, étaient aménagées deux guérites crénelées (Mahindad, 2002).

Après leur installation, les Français ont effectué quelques travaux de consolidation, de réparation et de réaménagement à l'intérieur du fort. Les réparations sont effectuées sur la porte d'entrée, la partie sud de la façade et sur certaines embrasures, par contre la consolidation était plutôt à l'intérieur du fort, alors que le réaménagement s'est fait en divisant la hauteur de la grande salle latéralement en deux pour former un rez-de-chaussée qui abrite un magasin de stockage des liquides et un premier étage réservé pour un magasin de farine relié entre eux par un monte-charge.
En 1980, le consultant de l'UNESCO dans son rapport d'écrit l'état du bordj Moussa à l'intérieur, «les locaux sont encombrés de gravois et les sous-sols ne sont pas accessibles. D'immenses salles voûtées pourraient être facilement restaurées. La plus importante, située à l'entrée, retrouverait son volume initial si on supprimait le plancher intermédiaire construit au siècle dernier. En revanche, pour d'autres salles, il sera nécessaire de reconstruire les planchers.» ( Herrmann ,1980).

En 1989, après quelques travaux de réhabilitation le fort est restitué à la ville, fut utilisé en musée. Les travaux de réhabilitation ont altéré la typologie architecturale du fort sans pour autant répondre aux normes d'expositions muséales internationales.

Actuellement, le fort est toujours réutilisé comme musée. Il a gardé l'enveloppe originale de l'édifice espagnol, avec des murs épais. Néanmoins certains aménagements réalisés à l'époque française ont disparu comme le plancher qui divise la hauteur de la grande salle en deux. Le musée se développe sur plusieurs niveaux:

Un sous- sol s'étendant dans la partie sud du bâtiment et dans l'épaisseur, dont le seul accès se trouve au niveau du RDC.

Le rez-de-chaussée est composé de trois voûtes d'une hauteur de neuf mètres.

D'un niveau intermédiaire

D'un étage qui a été à l'origine une esplanade à l'époque espagnole, réaménagé après comme caserne qui se développe en deux niveaux par les français. Le deuxième niveau était supprimé lors des travaux de restauration de 1989. Le premier niveau abrite aujourd'hui les bureaux de l'administration du musée, à partir de ce niveau sont distribués les terrasses, le cavalier, et les guérites crénelés.

Les parois extérieures du bordj sont percées de plusieurs types d'ouvertures, on relève :

Sous forme d'arc plein cintre, constitué d'arcature composée de deux rangées de briques en alternance longitudinalement et transversalement et d'un jambage de même matériau. 
Sous forme d'arc surbaissé constitué d'arcature composée d'une rangée de briques en alternance longitudinalement et transversalement et d'un jambage de même matériau.

Des meurtrières sous forme rectangulaire avec une plate bande en pierre et un jambage en briques.

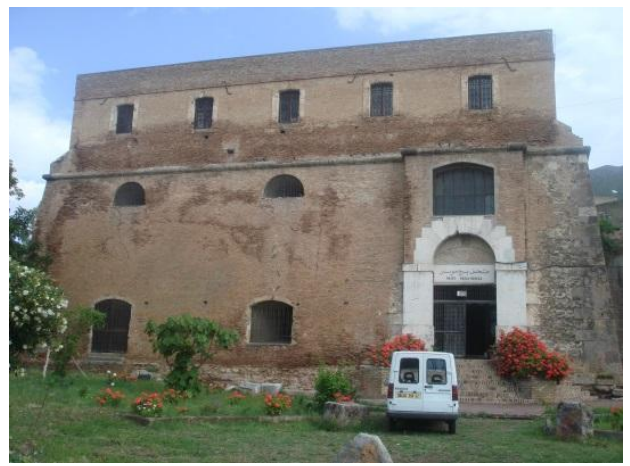

Fig. 7- vue sur la façade nord du bordj ( korichi, Amina 2010)

Concernant le système constructif, on relève trois types de murs porteurs :

Des murs porteurs double appareillés en grosses pierres quadrangulaires entrecroisées et superposées jusqu'à une hauteur de $80 \mathrm{~cm}$, en alternance avec des chaines de briques, dans les soubassements.

Des murs d'angles réalisés par assemblage de pierres quadrangulaires liés à sec (sans mortier).

Des murs porteurs appareillés entièrement en briques disposées sur champs liées par un mortier de chaux de sable, et mélangé avec gravier obtenue du broyage de la brique et de la tuile..

En 2009, le projet de restauration du fort Moussa a été lancé par la direction de la culture de Bejaia. Après les travaux de désherbages, les travaux de décapage des crépissages ont été effectués, le monument trouve enfin son authenticité.

Injecter des fonctions très légères au niveau des bastions comme des ateliers de musique pour enfants.

Aménagement d'un amphithéâtre en plein air.

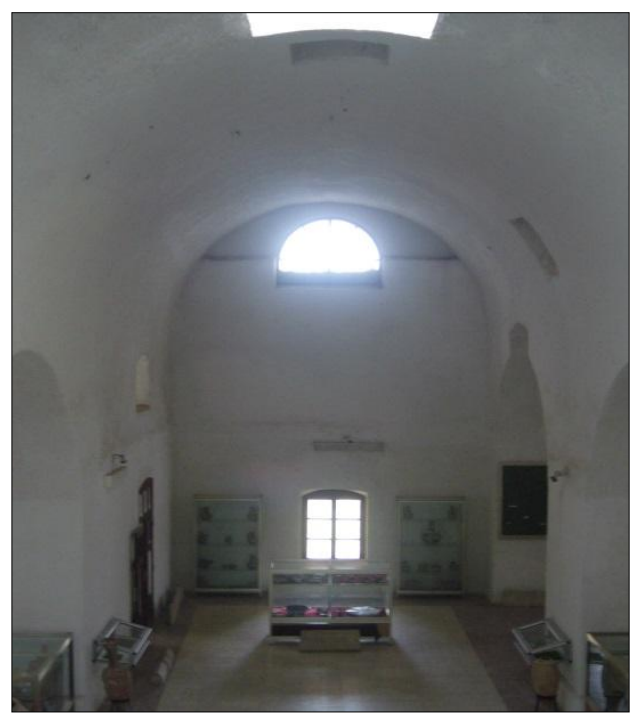

Fig. 8- vue sur la grande salle avant le décapage des crépissages (Korichi, Amina, 2008)

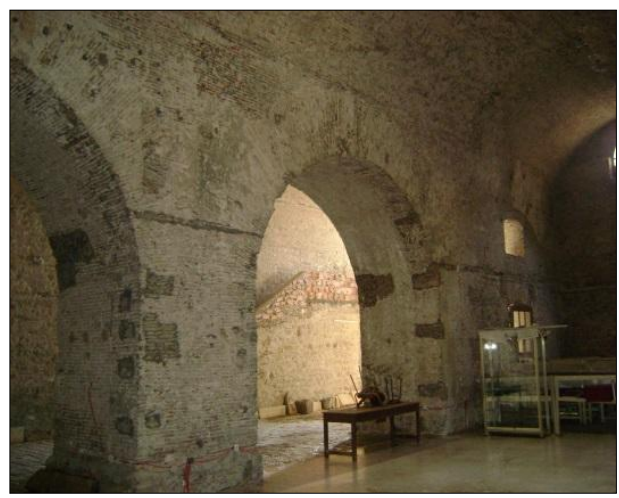

Fig. 9- vue sur la grande salle après le décapage des crépissages (Korichi, Amina, 2011)

\section{Le bordj Moussa}

C'est le seul monument réutilisé depuis 1989 en musée qui abrite une collection des restes archéologiques de l'époque romaine, et quelques tableaux de peinture qui ont été exposés au niveau de l'actuel siège de la radio Soummam. C'est par nécessité de trouver un endroit pour déplacer les objets collectionnés que la bordj fut réhabilité et réutilisé comme musée. Selon le responsable du musée les objets se perdent dans le volume du bordj, et la plupart des visiteurs sont impressionnés par le monument lui-même. 
Pour cela le problème qui se pose dans la réutilisation du bordj c'est bien au niveau de la forme/fonction. La surface, le volume, et l'éclairage ne s'adaptent nullement aux normes muséologiques.

On peut constater que ce monument a pu trouver une nouvelle fonction dans le domaine culturel, tout comme les monuments européens, cependant cette réutilisation, un peu forcée et non planifiée, ne procure pas les résultats escomptés. Ceci ressent sur sa fréquentation par le public. Avant sa fermeture en 2008, le musée était visité par le large public, les touristes, les groupes des scolaires, et les chercheurs, et sa fréquentation était plus accentuée pendant la période estivale. Le nombre des visiteurs pouvait atteindre mille visiteurs par mois pendant cette période. Pour les autres saisons ce sont beaucoup plus les groupes scolaires, et les étudiants chercheurs dans le domaine de l'archéologie et de l'histoire qui le fréquentaient.

Depuis sa réutilisation le musée n'abrite qu'une seule exposition permanente. L'absence d'animation et d'activités culturelles rend le monument figé dans son contexte et non rentable. Actuellement le fort est en cour de restauration, et sa réutilisation est un sujet de discussion entre la direction de la culture de Bejaia, le bureau d'étude chargé de sa restauration, les responsables du musée, et la public notamment par le biais des associations.

\section{Conclusion}

Bejaia possède un immense patrimoine défensif qui, malheureusement, est en train de dépérir au vu et au su de tout le monde. Des richesses irremplaçables de cette architecture militaire, qui sont en péril, la dégradation puis la disparition de leurs éléments les plus caractéristiques s'accélère dans l'indifférence quasi-totale.

A l'instar d'autres villes algériennes, l'absence de protection et de réutilisation des monuments historique est plus remarquable dans le cas de Bejaia. La liberté absolue de squatter les monuments défensifs et la possibilité incontrôlée de transformer et de détruire des bâtiments de l'architecture militaire d'époque coloniale, exprime très bien la défaillance de la politique patrimoniale suivie par les institutions locales en charge du patrimoine.

Le patrimoine défensif présente certaines spécificités liées à sa nature et à son rôle qui a généré des formes et espaces pas toujours faciles pour de nouveaux réaménagements. C'est également un patrimoine puissant accompagné par des exigences d'intendance. Dans le cas de Bejaia, les monuments défensifs classés sont pris comme tous les autres monuments du parc patrimonial, il n’y a pas de spécificité pour cette architecture militaire. La réutilisation de ces monuments se fait donc sans prendre en compte leur spécificité architecturale, et leur fonction d'origine. Cette indifférence engendre toutes sortes de mutilations et de dégradations irréversibles à notre patrimoine militaire.

\section{References}

Khelifa A. (2007). Histoire d'EL DJAZAIR, éditions Dalimen, p. 55.

Epalza M, Vilar J. (1988). Plans et cartes hispaniques de l'Algérie de XVIème au XVIIIème siècle, édition France, volume I, p : 129.

Dehabsourg L. S. (1999). Bougie la perle de l'Afrique du nord., L'hamattan, Paris, p. 37.

Feraud Ch. (2001). Histoire de bougie, édition Bouchene, p. 79.

Valerian D. (200). Bougie, port maghrébin à la fin du moyen âge (1067-1510), thèse pour obtenir le grade de docteur de l'université Paris 1, p. 54.

Mr Khelifa, Rapport de la mission effectué dans la ville de Bejaia et de Tizi Ouzou du 9 février au 11 février 1970.

Srira Zitouni et Marie- Colette Dépierre, Rapport sur la mission effectuée à Bejaia du 9 au 12 avril 1978.

Korichi A. (2011). La sauvegarde et la réutilisation des monuments du système défensif de la ville de Bejaia. Mémoire de magister, UMMTO, p. 102. 
Korichi A. (2011). La sauvegarde et la réutilisation des monuments du système défensif de la ville de Bejaia. Mémoire de magister, UMMTO, p. 102.

Féraud CH. (1868). Occupation de Bougie, Revue Africaine N :3, p. 50-51.

Korichi A. (2011). La sauvegarde et la réutilisation des monuments du système défensif de la ville de Bejaia. Mémoire de magister, UMMTO, p. 145.

Korichi A. (2011). La sauvegarde et la réutilisation des monuments du système défensif de la ville de Bejaia. Mémoire de magister, UMMTO, p. 147.

Epalza M, Vilar J. (1988). Plans et cartes hispaniques de l'Algérie de XVIème au XVIIIème siècle, édition France, volume 349.

Ministère de la guerre. (1838). Tableau de la situation des établissements français dans l'Algérie, imprimerie impériale, paris p. 99.

Mahindad N. (2002). Essai de restitution de l'histoire urbaine de la ville de Bejaia, thèse magistère EPAU, p. 110.

Herrmann R. (1980), plan de sauvegarde du centre historique de Bejaia, UNESCO, p. 5. 